\title{
Ny Karazana Aretina Mpahazo Ny Olon-Dehibe Sy Ny Tanora Ao Amin'Ny Tananan'Ny Sahamamy Kaominina Mahavelona Toamasina II
}

\author{
Harison Rabarison and David Ramanitrahasimbola
}

\section{Fampidirana}

Sahamamy dia tanàna iray eo ho eo amin'ny 7-8 Km ao andrefan'ny tanànan'i Mahavelona (Longitida Atsinanana: 49 25' 39.2" - Latitida atsimo : 170 43' 53.1" - haavo: 31 $\mathrm{m})$, manamorona ny alan' ny "ANALALAVA" izay misy mponina mivelona amin'ny fambolena amin'ny ankapobeny. Avy amin'ny foko Betsimisaraka ny akamaroan'ny mponina ao aminy, ary miankina betsaka amin'ny ala sy ny voly an-koraka ny fiainany.

Mba hahafantarana ny fifandraisan'ny zavamaniry sy ny olona sy mba hampisehoana ny mahazava-dehibe ny ala amin'ny fiainan'ny mponina dia nanao fanadihadiana tany an-toerana ny ekipan 'ny MBG (Missouri Botanical Garden) sy ny KNARP (Karoka Nasionaly Ampiharana ny Raki-Panafody). Fanadihadiana izay tafiditra indrindra tao antin'ny atrik'asa momban'ny fifandraisan'ny zava-maniry sy ny olombelona (Ethnobotanique) ary novatsian'ny ICBG (International Cooperative of Biodiversity Group) tarihin'i Pr David Kingston vola. Ny valin'ity fanadihadiana ity sy ireo fanadihadiana hafa izay samy mikasika ny fifandraisan'ny olombelona sy ny zavamaniry avokoa dia havondrona ho antotan-taratasy ho fandresen-dahatra ny manam-pahefana isan-tsokajiny sy ny mponina manodidina amin'ny fikajiana sy fiarovana ny alan' "Analalava".

\section{Tanjona}

Fahafantarana ny fampiasana ny zavamaniry amin'ny fikojakojana ny fahasalamana, indrindra eo amin'ny olondehibe sy ny tanora.

\section{Fomba Fiasa}

Mba hahatratrarana ny tanjona napetraka etsy ambony dia nisy ny fomba nentina nanantanteraka ny fanadihadiana. Voalohany dia raha tonga tao an-tanànan'ny Sahamamy izahay dia nisy ny fanazavana ny anton-dia tamin'ireo mponina. Voalaza tamin'izany fa ny fanadihadiana tiana hatao dia mikasika indrindra ny aretina mpahazo ny olona ao an-tanàna sy ny zavamaniry entin-drizareo manasitrana ny aretina. Ireto manaraka ireto ny karazam-panontaniana nentina naka ny tahirin-kevitra avy tamin'ny mponina tamin'izany:

- Inona avy ny aretina mpahazo ny olon-dehibe sy ny tanora?

- Ahoana ny fomba fitrangan 'ny aretina?

- Inona ny antony mahatonga azy?

- Oviana ny fotoana fisehoan 'ny aretina?

- Inona ny karazan-javamaniry fampiasa hitsaboana azy?

- $\quad$ Aiza ny toerana hakana sy hanirian'ireo zavamaniry fampiasa?

- Inona ny singa ampiasaina avy aminy (raviny sa tahony sa fakany)?

- $\quad$ Ahoana ny fomba fikarakarana azy?

- Ahoana ny fatrany sy ny fomba ampiasana azy?

- Maharitra hafiriana ny fitsaboana?

- Ahoana ny habetsak ilay zavamaniry?

\section{Correspondence}

Harison Rabarison, Conservation International-Madagascar (Cl-mad), BP 5178, Antananarivo 101, MADAGASIKARA.

hrabarison@conservation.org

David Ramanitrahasimbola, IMRA, BP 3833, Antananarivo101, MADAGASIKARA.

hasimbola67@yahoo.fr

Ethnobotany Research \& Applications 3:359-362 (2005) 
Tabilao 1. Ireo aretina mpahazo ny olon-dehibe sy tanora ary ny fomba fisehony.

\begin{tabular}{|l|l|}
\hline Aretina & Famantaran'ny mponina azy \\
\hline Tazo & $\begin{array}{l}\text { mangetaheta vao maraina, malemilemy, mangan-doha, mafaitra ny vava, mafana hoditra, mandoa } \\
\text { ranon `afero, mafana fofon aina ary mangovitra. }\end{array}$ \\
\hline $\begin{array}{l}\text { Haronjanana } \\
\text { na harerahana } \\
\text { na Hozatra }\end{array}$ & $\begin{array}{l}\text { mangotsikotsika, manakotsako ny vanin-taolana, manevineviky, henjana ny vantana, tsy avy mi- } \\
\text { aina ary tsembotsemboka. }\end{array}$ \\
\hline Mivalan-dra & marary kibo ary mangery rà (karazana valan'aretina). \\
\hline Vay vonto & mivonto kely sady mafimafy ary mangidihidy dia mihabe ny vodiny ary foy ny lohany. \\
\hline Kohaka & mangitikitika ny tratra, sempotra ary mikohoka. \\
\hline Solopiso & mamaivay be ny mahalehilahy, misy mivontovonto indraindray ary tsy afaka mamany tsara. \\
\hline Angorohosy & Tsy afaka miaina tsara, misy hatsaka mavomavo ny maso ary hatsatra ilay olona. \\
\hline
\end{tabular}

Rehefa vita moa ny fametrahana ny fanontaniana dia nijery ny toerana misy ireo zavamaniry mba nahafahana naka santionany izay nahafahana namatatra ny anarany ara-tsiansa. llaina ity famantarana anarana siantifika ity satria fanadihadiana siantifika no natao.

\section{Vokatra}

Ny vokatra azo tamin'ny fanadihadiana dia nofintinana amin'ireo tabilao roa etsy ambany. Ny Tabilao 1 dia mampiseho ireo aretina mpahazo matetika ny olon-dehibe sy ny tanora ary ny fomba fitragany. Ny Tabilao 2 dia mampiseho ny famintinana ny fitsaboan'ny olona ny aretina mpahazo azy ireo ary ny Tabilao 3 dia mampiseho ny toerana hanirian ireo zavamaniry ireo sy ny toetoetrany.

\section{Fanazavana}

Ireto misy fanamarihina vitsivitsy mifanadrify amin'ny tabilao etsy ambony. Ny ravina sy taho dia maromaro ny habetsahany ilaina amin `ny ankapobeny. Misy karazan-kazo hafa koa azo atao fanafody harerahana toy ny harongana
(Harungana madagascariensis) izay ny hodiny no ampiasaina amin'izany. Miisa enina ambinifolo (16) karazana eo ho eo ireo zavamaniry ampiasain'ny mponina amin'ny fitsaboana ny aretina mpahazo ny olon-dehibe sy ny tanora.

\section{Famintinana}

Ity vokatra azo ity dia manambara fa saro-bidy ho an 'ny mponina manodidina ny alan' "ANALALAVA" ny zavamaniry amin'ny ankapobeny satria na tsy manam-bola aza ny mponina tantsaha dia afaka mahita eo an-toerana ny zavatra ilainy amin`ny fikojakojany ny fahasalamany.

\section{Fisaorana}

Fisaorana lehibe no atolotra ireo rehetra nandray anjara (ireo mponina rehetra ao Sahamamy) sy andriamatoa Donnat, mpitsabonetim-paharazana ao Sahamamy tamin'ny nahavitana izao fanadihadiana izao.

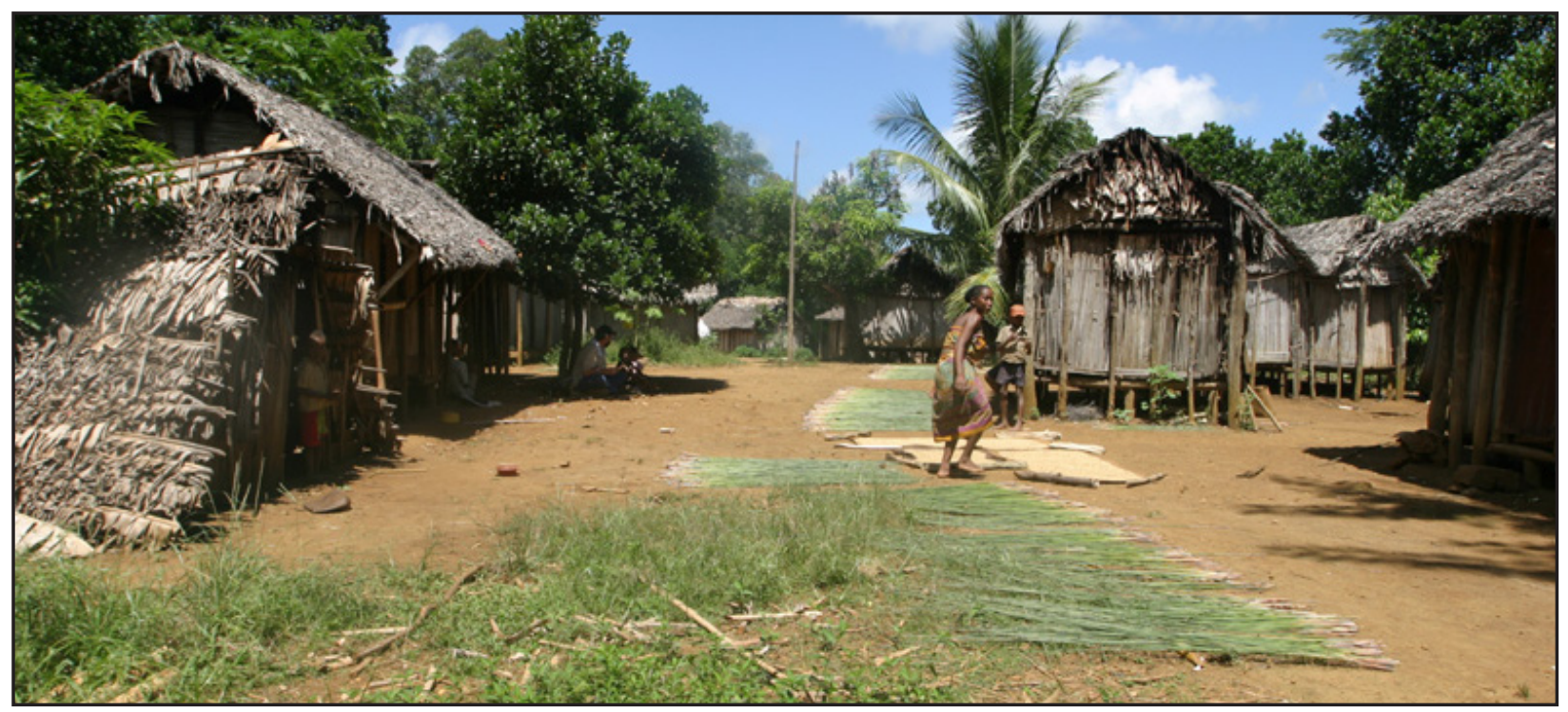

www.ethnobotanyjournal.org/vol3/i1547-3465-03-359.pdf 


\section{Rabarison and Ramanitrahasimbola - Ny Karazana Aretina Mpahazo Ny Olon-Dehibe Sy Ny Tanora Ao Amin'Ny Tananan'Ny Sahamamy Kaominina Mahavelona Toamasina II}

Tabilao 2. Fomba fikarakarana sy ny singa ampiasaina amin'ireo zavamaniry ampiasain'ny olona amin'ny fitsaboana.

\begin{tabular}{|c|c|c|c|c|}
\hline Aretina & Zavamaniry & Singa ampiasaina & Fikarakarana & $\begin{array}{l}\text { Fomba fampiasa sy } \\
\text { fatrany }\end{array}$ \\
\hline \multirow{4}{*}{$\begin{array}{l}\text { Tazo (Fotoana } \\
\text { mangatsiaka vo- } \\
\text { lana jona, jolay ary } \\
\text { aogositra) }\end{array}$} & $\begin{array}{l}\text { Tongo-borona } \\
\text { Cabucala sp. }\end{array}$ & \multirow[t]{3}{*}{ Raviny sy tahony } & \multirow[t]{3}{*}{$\begin{array}{l}\text { Tenehina atao maty } \\
\text { sasaka }\end{array}$} & \multirow[t]{3}{*}{$\begin{array}{l}\text { Nomina matetika man- } \\
\text { drapahasitrany }\end{array}$} \\
\hline & $\begin{array}{l}\text { Famahotrakanga } \\
\text { Lygodium sp. }\end{array}$ & & & \\
\hline & $\begin{array}{l}\text { Hazomamy } \\
\text { Bathiorhamnus sp. }\end{array}$ & & & \\
\hline & $\begin{array}{l}\text { Hazoambo } \\
\text { Xylopia sp. } \\
+ \\
\text { Vahizato } \\
\text { Secamone sp. }\end{array}$ & Raviny sy tahony & $\begin{array}{l}\text { Tenehina atao maty } \\
\text { sasaka }\end{array}$ & $\begin{array}{l}\text { Nomina in-telo isanan- } \\
\text { dro }\end{array}$ \\
\hline \multirow{4}{*}{$\begin{array}{l}\text { Haronjanana } \\
\text { na harerahana } \\
\text { na Hozatra } \\
\text { (Fotoana fiasana } \\
\text { horaka) }\end{array}$} & $\begin{array}{l}\text { Hazoambo } \\
\text { Xylopia sp. }\end{array}$ & \multirow[t]{3}{*}{ Raviny sy tahony } & \multirow[t]{3}{*}{$\begin{array}{l}\text { Tenehina atao very } \\
\text { sasaka }\end{array}$} & \multirow{3}{*}{$\begin{array}{l}\text { Nomina na giaina } \\
\text { Maraina, atoandro, } \\
\text { hariva (alohan`ny } \\
\text { sakafo) }\end{array}$} \\
\hline & $\begin{array}{l}\text { Tendrofony } \\
\text { Calantica sp. }\end{array}$ & & & \\
\hline & $\begin{array}{l}\text { Menakabonga } \\
\text { Combretum sp. }\end{array}$ & & & \\
\hline & $\begin{array}{l}\text { Elabaratra } \\
\text { Tachiadenus sp. }\end{array}$ & Singa rehetra & Tenehina & Nomina \\
\hline \multirow{2}{*}{$\begin{array}{l}\text { Fivalanan-dra } \\
\text { (valan`aretina) } \\
\text { (Fotoanan'ny ora- } \\
\text { na sy hafanana) }\end{array}$} & $\begin{array}{l}\text { Antafana } \\
\text { Terminalia catappa }\end{array}$ & Hodin`ny tahony & \multirow[t]{2}{*}{$\begin{array}{l}\text { Tenehina atao maty } \\
\text { sasaka }\end{array}$} & \multirow[t]{2}{*}{$\begin{array}{l}\text { Nomina na giaina rehe- } \\
\text { fa mangetaheta }\end{array}$} \\
\hline & $\begin{array}{l}\text { Goavitsina } \\
\text { Psidium cattleyanum }\end{array}$ & Raviny sy tahony & & \\
\hline $\begin{array}{l}\text { Vay vonto } \\
\text { (Mandava-taona) }\end{array}$ & $\begin{array}{l}\text { Tavolo } \\
\text { Tacca sp. }\end{array}$ & $\begin{array}{l}\text { Fakany na vodiny } \\
\text { (bulbe) }\end{array}$ & $\begin{array}{l}\text { Kinkisana amin `ny } \\
\text { vato ilay vodiny }\end{array}$ & $\begin{array}{l}\text { Sasana amin ny rano- } \\
\text { mafana ilay bay, hosor- } \\
\text { ana amin ilay faikany } \\
\text { rehefa foy ny lohany } \\
\text { dia bandina }\end{array}$ \\
\hline $\begin{array}{l}\text { Kohaka } \\
\text { (mangatsiaka) }\end{array}$ & $\begin{array}{l}\text { Vahivola } \\
\text { Paederia farinosa }\end{array}$ & Raviny sy tahony & Tenehina & $\begin{array}{l}\text { Nomina mandrapaha- } \\
\text { sitrana }\end{array}$ \\
\hline $\begin{array}{l}\text { Solopiso (MST) } \\
\text { (Mandava-taona) }\end{array}$ & $\begin{array}{l}\text { Famoha } \\
\text { Palmier indét. }\end{array}$ & Raviny & $\begin{array}{l}\text { Tenehina dia avela } \\
\text { hangatsiaka }\end{array}$ & Nomina \\
\hline \multirow[t]{2}{*}{ Angorohosy } & $\begin{array}{l}\text { Ambovahatra } \\
\text { Borreria verticillata }\end{array}$ & Raviny sy tahony & $\begin{array}{l}\text { Tenehina dia ham- } \\
\text { pangatsiahina }\end{array}$ & $\begin{array}{l}\text { Nomina in-telo } \\
\text { isan`andro }\end{array}$ \\
\hline & $\begin{array}{l}\text { Ambora be ravina } \\
\text { Burasseia sp. }\end{array}$ & $\begin{array}{l}\text { Raviny sy hoditrin'ny } \\
\text { tahony }\end{array}$ & Tenehina & Nomina \\
\hline
\end{tabular}


Tabilao 3. Toerana haniriany sy ny habetsahany ary ny toetoetran'ireo zavamaniry ireo.

\begin{tabular}{|l|l|l|l|}
\hline Zavamaniry & Karazany & Toerana & Habetsahany \\
\hline Tongoborona (Cabucala sp) & Hazo madinika & Ala & Maro \\
\hline Famahotrakanga (Lygodium sp) & Vahy & Ala, Roranga, Savoka & Maro \\
\hline Hazomamy (Bathiorhamnus sp) & hazo & Savoka sy ala & Antonony \\
\hline Hazoambo (Xylopia sp) & Hazo & Savoka sy ala & Maro \\
\hline Vahizato (Secanome sp) & Vahy misy ronono & Savoka & Maro \\
\hline Tendrofony (Calantica sp) & Hazo & Savoka sy ala & Maro \\
\hline Menakabonga (Combretum sp) & Vahy & Ala & Maro \\
\hline Elabaratra (Tachiadenus sp) & Bozaka & Savoka sy roranga & Maro \\
\hline Antafana (Terminalia catappa) & Hazo & Savoka sy ala & Maro \\
\hline Goavitsinà (Psidium cattleyanum) & Hazo madinika & Savoka sy roranga & Maro \\
\hline Tavolo (Tacca sp) & Bozaka & Ala sy roranga & Maro \\
\hline Vahivola (Paederia farinosa) & Vahy mantsina & Roranga & Maro \\
\hline Ambovohatra (Borreria verticillata) & Bozaka & $\begin{array}{l}\text { Savoka, roranga, } \\
\text { antanimboly ary antanana }\end{array}$ & Maro \\
\hline Famoha (Palmier indét.) & & Tanimboly sy roranga & Antonony \\
\hline Harongana (Harunga madagascariensis) & Hazo & Savoka, roranga ary tanimboly & Maro \\
\hline Ambora be ravina (Burasseia sp) & Hazo & Ala & Maro \\
\hline
\end{tabular}

